\title{
L'hospitalité : déconstruire le concept, décoloniser le sens
}

Renan Gonçalves Rocha (IFG)*

https://orcid.org/0000-0001-9837-5166

\section{Résumé :}

Dans cet article, nous allons faire un parcours au sein de la pensée derridienne de l'aporie. Il s'agit de montrer comment une approche aporétique de la question de l'hospitalité nous renvoie à la disruption du « concept ». Du fait de la construction d'un champ homogène du « sens » et de l'homogénéité du concept même, sa perspective monolingue, entendue comme la colonisation en tant que production de l'unicité du propre sens, n'ouvre pas de possibilité ambivalente au transbordement comme perspective de déconstruction. Alors que la déconstruction de l'homogénéité du concept est centrale parce qu'elle ouvre à d'autres perspectives, à d'autres sens et à d'autres concepts, c'est précisément cette démarche qu'emprunte l'hospitalité : une déconstruction du « concept » et une décolonisation du « sens ». Ainsi, si pour Derrida l'hospitalité est prise comme sujet de sa pensée, c'est parce que son injonction constitutive, son rapport au concept lui-même est aporétique, intrinsèquement ambivalent et disruptif. L'ouverture du " concept », c'est l'hospitalité.

Mot-clés : décolonial ; déconstruction ; concept ; sens ; hospitalité.

\section{Resumo:}

\section{Hospitalidade: desconstruir o conceito, descolonizando o sentido}

Neste artigo, faremos um passeio pelo pensamento derridiano da aporia. Trata-se mostrar como uma abordagem aporética da questão da hospitalidade nos remete à ruptura do "conceito". Devido à construção de um campo homogêneo de "sentido" e à homogeneidade do próprio conceito, sua perspectiva monolíngue, entendida como colonização e como produção da unicidade de seu próprio sentido, parece não abrir nenhuma possibilidade ambivalente, ao transbordamento como perspectiva da desconstrução. Se a desconstrução da homogeneidade do conceito é central porque abre outras perspectivas, outros significados e conceitos, é precisamente esta abordagem que a hospitalidade assume: uma desconstrução do "conceito" e uma

* Professor de Filosofia do Instituto Federal de Goiás - IFG e doutorando em Filosofia pela Universidade Paris Nanterre. Pesquisador ligado ao Núcleo de Pesquisa em Filosofia - NUPEFIL (IFG) e ao Institut de Recherches Philosophiques - IREPH (Université Paris Nanterre). E-mail: renangrocha@yahoo.com.br 
descolonização do "sentido". Assim, se para Derrida a hospitalidade é tida como tema de seu pensamento, é porque sua injunção constitutiva, sua relação com o próprio conceito é aporética, intrinsecamente ambivalente e disruptiva. A abertura do "conceito" é a hospitalidade.

Palavras-chave: Descolonização; Desconstrução; Conceito; sentido; Hospitalidade.

L'approche derridienne sur l'homogénéité du sens ou du concept en tant qu'« unité formelle de la forme et du sens » (DERRIDA, 1967, p. 13) s'exprime comme une imposition monolingue (DERRIDA, 1996), c'està-dire non seulement l'imposition d'une langue, mais aussi d'un sens homogène, d'une tradition, d'une mémoire, et d'un seul imaginaire possible pour les sens. Or, il s'avère que le concept entre en collision avec lui-même et c'est justement dans cette collision, que nous pouvons penser la centralité de l'hospitalité pour Derrida.

Une pensée aporétique du concept crée une tension dans la langue elle-même, en faisant glisser le sens du concept, en l'ouvrant à l'autre, à d'autres perspectives, d'autres mémoires, et d'autres traditions. Il s'agit de la disruption avec le monolinguisme, avec cette « unité » construite comme concept et comme production d'un sens uniforme. Ce glissement se produit par une sorte de jeu de position où les concepts n'ont plus de signifiant mais sont toujours performatifs. Ils glissent d'un signifiant à l'autre, ce qui déstabilise leur propre unité. En effet, c'est une sorte de traduction "intralinguistique » (RODRIGUES, 2020), qui va au-delà de la traduction d'une langue à l'autre : elle traduit la langue à elle-même, ce qui ne produit pas seulement une polysémie du concept mais aussi la déstabilisation du propre sens du concept.

Selon Derrida, l'imposition d'un sens par le concept n'est jamais une détermination absolue, sans possibilités d'échappa- toire. L'établissement de frontières ou d'un « champ » pour le concept ou pour le sens vient avec le transbordement de ces frontières imposées. Ainsi, si l'hospitalité est un exemple de l'aporie pour Derrida, c'est parce que l'accueil correspond au dépassement du concept d'accueil lui-même, puisqu'il est traduit différemment dans chaque cas. Cette traduction interne du concept envers lui-même qui déstabilise son sens, fait aussi que le concept présente d'innombrables implications, ainsi qu'une performativité toujours en déplacement.

\section{L'aporie : dépasser le concept dans le concept}

Un dépassement, un débordement n'implique-t-il pas déjà une limite à dépasser ? Et par conséquent, une nouvelle dualité entre la limite et son au-delà ? Nous retrouvons ici la porosité de la question du sens du concept et la complexité du langage derridien. En effet, le sortir (KOFMAN, 1983) ne signifie pas sortir d'une extériorité radicale mais plutôt se déplacer parmi les apories, créant constamment de nouvelles apories. Il est question d'une " capacité souvent tapie [...], de retourner les perspectives et d'extraire des forces actives depuis des lieux où elles étaient immobilisées, fixées, liées, piégées » (PROUST, 1997, p. 5-10).

Dans ce langage sinueux, il ne faut pas penser le débordement, l'au-delà, le dépassement, comme des bords à dépasser. Ces concepts apparaissent plutôt en mou- 
vement entre les pores perméables d'une peau limitante, comme pour se sortir d'embarras aporétiques paralysants. Finalement, la pensée derridienne de l'aporie ${ }^{1}$ ne se rapporte pas au débordement classique de la limite en tant qu'établissement de l'intériorité et de l'extériorité. Chez Derrida « l'impasse ou l'aporie tient au fait qu'il n'y a pas de limite. Il n'y a pas encore ou il n'y a que deux bords : la limite est trop poreuse, perméable, indéterminée »(DERRIDA, 1995, p. 44). L'au-delà, le débordement, est donc compris à partir de la perspective de « sortir de soi en sa position » (DERRIDA, 1968).

Ce postulat, déjà développé par Derrida dans ses écrits de 1968 (La différence), explique par exemple, la complexité de la discussion par rapport à la trace, et surtout comment cette proposition bouleverse le sens du concept à partir de cet au-delà-dans. Ce postulat explique également une prise aporétique de la fermeture du concept qui permet de sortir de l'impasse paralysante comme la fermeture même. La trace est, selon Derrida (1968), ce qui ne se laisse pas saisir dans le concept : elle est à la fois en dedans et en dehors du concept. En effet, la trace :

n'étant pas une présence mais le simulacre d'une présence qui se disloque, se déplace, se renvoie, n'a proprement pas lieu, l'effacement appartient à sa structure. Non seulement l'effacement qui doit toujours pouvoir la surprendre, faute de quoi ne serait pas trace mais indestructible et monumentale substance, mais l'effacement de lieu et la fait disparaître dans son apparition, sortir de soi en sa position (DERRIDA, 1968).

Dans L'écriture et la différence (1967), il présente déjà la notion de sortir de l'écriture à l'intérieur d'elle-même. C'est-à-dire la pensée d'une écriture qui se déplace «sur

1 Et, il faut y compris reconnaître l'influence de Sarah Kofman sur sa pensée. une ligne brisée » et qui brise aussi la promesse de la parole - le dire. Comme nous l'explique Jean Michel Salanskis :

l'événement du dire ne peut se protéger de sa résorption dans le Dit, c'est ce à quoi il arrive. La déstabilisation de la tendance substantialisante de la métaphysique, à laquelle Derrida est surtout attachée, doit donc être étudiée et espérée autant en référence à l'analyse du Dit comme trace [...] qu'à travers la revendication du Dire comme adresse [...]. (SALANSKIS, 2010, p. 111-112)

Il s'agit de dire en trahissant la promesse du vouloir-dire (DERRIDA, 1983, p. 36), une promesse déjà échouée. Plus tard, Derrida évoque dans En ce moment même (1998) la sortie de la langue : « le passage au-delà de la langue requiert la langue ou plutôt le texte comme lieu des traces pour un pas qui n'est pas (présent) ailleurs » (DERRIDA, 1998, p. 170). Et toujours pour réfléchir sur le concept et ses débordements, dans Mot d'accueil (1997), il prend l'exemple de l'ouverture dans le concept du politique, à savoir le politique au-delà de lui-même. D'où la notion du politique « au-delà du politique, mais dans le politique » (BENSUSSAN, 2018, p. 81). C'est une sorte d'excès du concept sur lui-même, un excès que Gérard Bensussan traduit comme une "scène de l'au-delàdans » (BENSUSSAN, 2018, p. 138). Bensussan explique que chez Derrida il existe une sorte de scène où il faut penser :

au-delà de l'éthique dans l'éthique, et au-delà de la politique dans la politique [...] C'està-dire [il faut tenter d'agir, nous dirait Derrida], sur une scène où l'au-delà se présente dans le lieu même censé le contenir et le nier et où, par conséquent, « il ne fait pas signe vers du non-politique ». Une scène dont les coulisses seraient incessamment ouvertes. [...] La scène qu'il figure, où les protagonistes peuvent être nombreux, aller et venir, discourir et se taire, offre la figuration 
dramatique de notre monde, de notre situation dans ce monde cassé [...] (BENSUSSAN, 2018, p. 86).

C'est donc dans ce langage sinueux de l'aporie que l'au-delà et le débordement ne sont pas envisagés à partir de l'idée de bords à dépasser mais comme une porosité où se trouve l'ouverture du concept et la déstabilisation d'un sens monolingue du concept. Pour expliquer cet excès à partir de l'aporie, Derrida illustre encore dans son ouvrage Mot d'accueil, avec le concept de paix, ce que Bensussan a nommé la scène de l'au-delà-dans. Cette notion est décisive pour décrire les implications de la réflexion derridienne sur le concept et la formation d'un sens homogène, à savoir le monolinguisme intrinsèque à une perspective coloniale, qui s'impose en tant que concept mais qui impose surtout les sens possibles de toute la conceptualité. Ainsi, l'effort de la déconstruction de l'unicité du concept va de pair avec la décolonisation du sens, autrement dit, avec l'ouverture radicale à d'autres possibilités de sens et de concepts. Selon Derrida (1996-1997, f. 5) l'hospitalité est cette déconstruction dans tout concept, pas juste parce qu'elle déboite cette violence du comprendre et du saisir conceptuel mais surtout parce qu'elle est l'ouverture à l'incompréhensible. En tant qu'ouverture radicale, elle est aussi l'ouverture d'un concept à l'autre, et même à son contraire, comme une cohabitation de l'un dans l'autre.

La notion d'hospitalité chez Derrida est ainsi utilisée pour expliquer l'enclavement dans le concept, et le dépassement du sens du concept, là où l'ouverture hospitalière à l'autre est déjà la déstabilisation de l'homogénéité du sens. Le propre concept d'hospitalité s'ouvre au-delà de lui-même, et ainsi, si chaque concept est ouvert à l'autre, comme hantise de l'un sur l'autre, l'autre est déjà au-dedans du concept, il y est enclavé dans l'autre. L'hospitalité comme cette expérience d'ouverture déplace, disloque, l'unicité de la forme et dégage une pensée du concept avant tout comme l'expérience avec l'autre.

L'hospitalité comme cette expérience déconstructrice n'est pas uniquement un exemple parmi d'autres, elle est l'exemple de la déconstruction par tous les entrelacements et les apories qu'elle implique - la sortie de soi en sa position, le dépassement $d u$ concept dans le concept - déconstruit le lieu lui-même du concept et de son intentionnalité. Ainsi selon Derrida, en tant que déconstruction du concept de concept, l'hospitalité s'ouvre à son autre jusqu'à la désidentification, là où l'autre dans cette relation est déjà tellement au-dedans qu'il désidentifie le propre concept lui-même.

La pensée de l'hospitalité comprend à la fois l'entrelacement et l'enclavement, là où le dedans est déjà entrelacé au dehors. C'est pourquoi la topologie du concept, son cadre, son sens, sont déstabilisés, voire contaminés, par ce qui se trouve en-dehors du concept. C'est le sens même d'homogénéité, de monolinguisme, qui se trouve en danger. C'est ce que nous pouvons observer aussi dans la compréhension derridienne du concept de paix, qui est une manière de penser les concepts en général. Il dit :

[le concept de paix] garde une part politique, il participe du politique même si une autre part en lui dépasse un certain concept du politique. Le concept s'excède lui-même, il se déborde, autant dire qu'il s'interrompt ou se déborde pour former ainsi une sorte d'enclave au-dedans et au-dehors de lui-même : 'au-delà dans'. (DERRIDA, 1997a, p. 146)

Cette notion de l'au-delà dans qui est décisive pour décrire les implications de la réflexion derridienne, est la scène du concept 
et la scène d'enclavement ininterrompue qu'est l'hospitalité. Néanmoins, l'hospitalité est aussi un concept qui implique de remettre en question le cadre conceptuel des lieux : la ville, la souveraineté, le territoire, le chez-soi, le corps, etc.

\section{Le lieu comme lieu du concept}

Pour repenser le concept d'hospitalité, Derrida repositionne alors la perspective ontopologique de la conceptualisation et de la détermination de l'espace. C'est-à-dire que le projet derridien est la remise en cause du lieu, de toute sa stabilité et sa sédentarisation, à partir du lieu du concept.

Il essaie de dépasser la détermination du lieu comme quelque chose d'homogène, de facilement identifiable, de fixe, à partir du concept lui-même qui est d'après lui instable. C'est précisément ce qu'il nomme comme " la différence locale » (CIXOUS, 2019), à savoir l'ouverture d'une place dans le lieu là où « la dis-location [du concept serait] toujours déjà dedans » (CIXOUS, 2019). Nous pouvons par conséquent penser l'effet de cette dis-location du concept de lieu comme un mouvement qui réintroduit et qui ouvre une place dans la compréhension de la localité elle-même. Il dégage un mouvement questionnant la rigidité du topos, le faisant entrer en collision avec lui-même. Autrement dit, il ouvre une place à un autre lieu dans le lieu, ainsi l'accueil, le chez-soi, peuvent être questionnés à partir de cette dis-location toujours déjà au-dedans du lieu. Dans Spectre de Marx (1997), cette problématique est amenée dans les termes suivants :

[...] Le processus de la dis-location n'en est pas moins archi-originaire, c'est-à-dire aussi « archaïque » que l'archaïsme qu'elle déloge depuis toujours. Il est d'ailleurs la condition positive de la stabilisation qu'il relance tou- jours. Toute stabilité en un lieu étant une stabilisation ou une sédentarisation, il aura bien fallu que la différance locale, l'espacement d'un dé-placement donne le mouvement. Et donne place et donne lieu. Tout enracinement national, par exemple, s'enracine d'abord dans la mémoire ou l'angoisse d'une population déplacée - ou déplaçable. "Out of joint" n'est pas seulement le temps, mais l'espace, l'espace dans le temps, l'espacement (DERRIDA, 1997, p. 137).

Penser cette dis-location du lieu à partir du lieu du concept, soit la déstabilisation du lieu par la différance, revient aussi à penser les conséquences de la philosophie de la différence en tant que philosophie de l'hospitalité déstabilisant des concepts comme ceux « de l'État-nation, de la souveraineté, des frontières, du sol et du sang ». Voilà l'ontopologie, comme une sorte d' « axiomatique liant indissociablement la valeur ontologique de l'être-présent (on) à sa situation, à la détermination stable et présentable d'une localité (le topos du territoire, du sol, de la ville, du corps en général) » (DERRIDA, 1997, p. 137).

La réflexion aporétique est là justement pour donner corps et mots à l'opération déconstructrice du lieu. Nous pouvons donc penser en termes de dé-placement du lieu et par conséquent remettre en question les lieux déterminés comme un chez-soi : la ville, la maison, le corps. C'est-à-dire l'espace dans la différence, voire la différance par rapport à lui-même.

\section{La tension sur la forme}

La remise en cause de la stabilité du concept, ou du sens homogène établi par l'imposition monolingue, se fait là où les limites de celleci se révèlent d'une part, et d'autre part lorsqu'il y a renvoi des limites du concept vers lui-même. Si la forme du concept est cette « 
unité formelle de la forme et du sens » (DERRIDA, 1967, p. 13), ce qui donne l'espace du concept, son lieu, sa spatialisation, c'est aussi ce qui désertifie, et ne prend pas en compte les forces au sein du concept et au sein de ce sens.

C'est pourquoi dans le texte Force et Signification (1967) ${ }^{2}$, il questionne la notion de force et forme pour penser le concept. En effet, la force est pensée comme une tension sur la forme, et comme une tension sur la forme du concept de force lui-même. Or, il renvoie le concept sur sa propre forme. Ainsi, la force « [...] est tension de la force elle-même [et] la forme fascine quand on n'a plus la force de comprendre la force en son dedans [...] » (DERRIDA, 1967, p. 11) ${ }^{3}$.

Donc d'après Derrida, la forme, ou le concept rigide et son sens, est un « parcours sur plan et plus librement [un] champ déserté de ses forces. Totalité désertée de ses forces même si elle est totalité de la forme et du sens, car il s'agit alors du sens repensé dans la forme » (DERRIDA, 1967, p. 13). Il ajoute : « un peu comme l'architecture d'une ville

2 Dans ce texte que nous pouvons comprendre, grosso modo, en deux parties, il présente d'abord le structuralisme et ses liens avec une sorte de platonisme. C'est dans la deuxième partie du texte qu'il discute deux exemples centraux pour notre réflexion, à savoir, une certaine compréhension du performatif et du géométrique, qui sont des notions au cœur d'une discussion de l'espace et du temps.

3 Derrida Jacques, L'écriture et la différence, Paris, France, Éd. du Seuil, 1967, p. 11. « Comprendre la structure d'un devenir, la forme d'une force, c'est perdre le sens en le gagnant. Le sens du devenir et de la force, dans leur pure et propre qualité, c'est le repos du commencement et de la fin, la paix d'un spectacle, horizon ou visage. En ce repos et en cette paix, la qualité du devenir et de la force est offusquée par le sens même » (Ibid.p. 44). «Il faut viser une certaine impuissance du langage à sortir de soi pour dire son origine, et non la pensée de la force. La force est l'autre du langage sans lequel celui-ci ne serait pas ce qu'il est ». Ibid. inhabitée ou soufflée, réduite à son squelette par quelque catastrophe de la nature ou de l'art. Ville non plus habitée ni simplement délaissée mais hantée plutôt par le sens et la culture » (DERRIDA, 1967, p. 13)

Cet exemple, qui n'est pas un exemple aléatoire, nous renvoie à une remise en question spatio-temporelle du concept, de son sens lui-même ou de l'espace inclus dans ce concept. La détermination de la forme rigide du concept en tant qu'espace architectural désertifié par quelque chose qui est survenu (comme une catastrophe), montre une détermination (même si désertique) des limites, des sens, des champs et des espaces du concept. La forme du concept montre qu'il existe une détermination spatiale et temporelle du concept et du sens monolingue.

En effet, il y a, bien entendu, la prise en compte de l'espace et du temps comme concepts pour eux-mêmes. Chez Derrida, il $\mathrm{y}$ a déconstruction du concept, et il y a donc aussi une déconstruction de l'espace et du temps et du propre sens imposé. La dimension architecturale, autrement dit le squelette de cette ville inhabitée, est remise en question à partir de cette tension autour de la forme et de cet espace sans vie.

Derrida réfléchit sur la « représentation mathématico-spatiale » (DERRIDA, 1967, p. 34) du concept et notamment du concept de lieu, qui implique ce sens univoque de pensée et surtout envisage la perspective coloniale dans son imposition monolingue. En synthèse, il s'agit de l'engagement remettant en cause le lieu à partir du lieu du concept, pour déstabiliser l'homogénéité du sens imposé.

\section{Aporie et différance}

Au même moment, la déconstruction du lieu du concept déplace, voire brise la no- 
tion de sens elle-même. Penser la différance dans le lieu en tant qu'espacement signifie déplacer la perspective du lieu lui-même. Il s'agit de déconstruire les références homogènes de l'espace pour ouvrir à d'autres références et d'autres perspectives. Cette ouverture, en tant que différance locale, est pensée à partir de la différence, c'est-à-dire du concept de différence remis en cause par la différance.

La substitution du « $e$ » par le « $a$ » a pour but de différencier la différence d'ellemême. L'idée est de provoquer, ou plutôt de forcer la rupture de sa cohésion. L'écriture de la "différance ", c'est un geste qui dévoile le mouvement de différenciation de la différence : cette greffe du « $a$ » dans la différence change la différence, déstabilise son homogénéité, la déplace et l'ouvre.

En somme, la substitution muette du « $e$ » en « $a$ » a pour but la différenciation : la différence n'est plus identique à ellemême. Elle est entraînée dans une chaîne de déplacements qui la lance hors de soi en soi. Autrement dit, c'est le mouvement qui « se disloque sans cesse dans une chaîne de substitutions différentes » (DERRIDA, 1968, p. 65).

Derrida propose une pensée aporétique de la différence, où ce déplacement dans le propre concept déstabilise l'homogénéité et sa détermination, entendues comme identiques en soi. C'est précisément un déplacement à l'intérieur du concept lui-même. La différance amène aussi un mouvement de déformation du concept, que nous pouvons penser " hors de ses gonds » (DERRIDA, 1997, p. 137), comme une disruption dans le concept. Ce geste dans l'écriture montre l'ouverture et le déplacement du concept.

Si l'hospitalité est chez Derrida un exemple majeur de l'aporie et de la déstabilisation du sens, ou encore du sens homo- gène du sens, c'est parce que l'hospitalité est toujours en déplacement. Il s'agit d'une proposition conceptuelle fuyante, qui comporte l'ambiguïté de la non-hospitalité, mais surtout de la tension à l'intérieur de sa propre forme conceptuelle. Elle est issue de la déconstruction du lieu du concept, c'est-à-dire de la remise en question et de l'ouverture du concept de l'hospitalité. Derrida pense l'hospitalité dans son identité non-identique. L'hospitalité dans sa différence avec elle-même, l'hosti-pitalité. Une formule de l'hospitalité à partir d'une philosophie de la différance. Un geste d'écriture permettant de dégager l'hospitalité de la stabilité du sens existant dans les vocabulaires juridico-politiques tout autant territoriaux, qu'éthiques et coloniaux.

Dans cette perspective, nous pouvons repenser le concept d'hospitalité pour pouvoir comprendre comment Derrida formule le déplacement d'un sens, à partir de la substitution à l'intérieur du concept. C'est exactement ce qui nous permet de penser sa manière d'expliquer l'artificialité de la construction du sens et ainsi l'artificialité de la déstabilisation de ce propre sens construit. La substitution dans l'hospitalité, nous montrera Derrida, déplace le propre sens du concept. Cela veut dire que l'imposition de l'imaginaire de réflexion, la mémoire que porte ce concept, est aussi déplacée dès que se change artificiellement l'imposition d'un sens par cette construction imposée. L'hospitalité est ainsi cette déstabilisation du propre sens à partir de la substitution au-dedans du concept. Autrement dit, c'est l'ouverture aux imaginaires et aux mémoires qu'impliquent le changement du sens du concept.

Le concept d'hospitalité est présenté ainsi chez Derrida comme une question non-déterminable, dépossédée d'un sens 
identique en soi. Il nous est donc impossible selon Derrida de savoir, comprendre, désigner, conceptualiser ou même légiférer sur l'hospitalité sans qu'elle devienne avant tout hosti-pitalité. Néanmoins, lorsque nous lisons les textes de Derrida sur l'hospitalité, nous relevons aussi la question de l'héritage des concepts et la possibilité du déplacement du concept lui-même, de sa tradition et de ses remises en question. Nous pouvons alors nous interroger sur les questions de la tradition et sur la tradition du concept, ce qui forme un sens. Dans cette perspective il faudrait donc déplacer et substituer la forme même du sens, ou traduire le sens en lui-même, qui change le sens propre et l'unicité du concept. Qu'est-ce que cela veut dire ? D’après Derrida, la substitution serait traduire et retraduire le concept pour lui-même, déplacer le sens et produire de la tension dans le sens, là où la substitution est l'épreuve de l'artificialité de la remise en question de la forme du sens, ou de l'imposition d'un sens, parce qu'elle impose artificiellement un déplacement dans le sens qui déstabilise l'unité du propre sens.

\section{Differance et hosti-pitalité}

La différance et l'hosti-pitalité sont des formes de greffe, ce sont des substitutions qui déplacent et ouvrent la différence et l'hospitalité à d'autres questions et perspectives. Il s'agit d'une action artificielle qui change la forme, le sens homogène du concept.

Ces deux concepts, selon Derrida, sont ainsi placés dans un jeu de substitutions qui déplace le sens. Derrida essaye de cette manière de déployer un mouvement où la substitution déplace les concepts et son sens. Cela implique donc de substituer le sens au-dedans du concept, là où le propre champ conceptuel est mis en question, c'est- à-dire en déplaçant le sens et la perspective imposée par son sens.

La substitution en tant que déplacement du sens crée une autre chaîne de sens, d'imaginaires, de concepts et de perspectives. Voilà le sens même, univoque, déstabilisé. En somme, dès lors qu'il y a une substitution dans le concept, il y a aussi une dislocation et un déplacement du sens lui-même. C'est par ce mouvement que nous ouvrons la question de l'hospitalité au-delà de l'hospitalité dans l'hospitalité.

Chez Derrida, c'est dans le mouvement de substitution que s'ouvre une possibilité d'interroger l'hospitalité dans sa performativité conceptuelle, anthropologique, dans la scène anthropocentrique d'une hospitalité toujours offerte - à qui ? Pouvons-nous ainsi déplacer l'hospitalité d'elle-même et parler d'une autre hospitalité ?

Dans cette perspective, la déstabilisation du sens du concept, du cadre imposé, remet justement tous les sens en question. C'est là, dans ce dépassement du sens du concept, du lieu, de la langue, que Derrida envisage le lien entre la déstabilisation du concept et la propre hospitalité, non seulement comme un exemple parmi d'autres mais comme une déstabilisation de l'homogénéité imposée du propre « concept » et de son cadrage homogène. Cette transgression est transgression de l'accueil, déplacement du concept comme déplacement de l'hospitalité et de son sens.

Ce n'est que dans cette scène que nous pouvons revenir au propre de l'hospitalité, c'est-à-dire que l'ouverture du propre sens, le dépassement du monolinguisme est l'hospitalité comme disruption de la perspective coloniale. L'hospitalité à tout autre est surtout et centralement une aporie disruptive. En d'autres termes, l'aporie disruptive dans le concept, c'est l'hospitalité. 
Ainsi, pour conclure, la substitution dans le concept ouvre la question de l'hospitalité à d'autres perspectives, dès lors qu'elle ouvre le propre sens du concept. Pour nous, la formulation du cadre monolingue (DERRIDA, 1996) est attachée à une perspective de constitution du sens avec toutes les implications nécessaires à la formulation d'une politique identitaire de formations $\mathrm{du}$ territoire, du « moi », de « l'autre » comme imposition coloniale. L'identité du concept et du sens, est la base de la propre identité, comme une construction, voire une imposition sur l'autre. C'est l'imposition du monolinguisme sur l'autre, ainsi, le déconstruire, c'est aussi le décoloniser.

La substitution dans le concept déplace le sens comme une façon de sortir du concept dans le concept - sortir du monolinguisme. La déstabilisation de cette perspective est ce qui permet une possibilité de penser autrement le propre concept et de l'ouvrir à d'autres imaginaires, à d'autres mémoires et à d'autres notions. Déconstruire le concept est une façon de décoloniser le sens.

\section{Références}

Bensussan Gérard, « La scène de l'au-delà-dans. Penser la politique avec Levinas et Derrida » in Derrida-Levinas : an alliance awaiting the politicalune alliance en attente de politique, édité par Orietta Ombrosi et Raphael Zagury-Orly et collaboration de Victoria Rehm, Philosophy, Italie, Mimesis International, 2018.

Cixous Hélène, Séminaire Des irréparables. IV. Le nom de l'Auteur ôté, Collège International de Philosophie, Fondation de l'Allemagne-Maison Heinrich Heine, 2018.
Derrida Jacques, Psyché, inventions de l'autre, Paris, France, Galilée, impr. 1998, 417 p.

Adieu : à Emmanuel Lévinas, Paris, France, Galilée, 1997a, 210 p.

Spectres de Marx : l'Etat de la dette, le travail du deuil et la nouvelle Internationale, Paris, France, Galilée, 1997, 278 p.

Le monolinguisme de l'autre : ou la prothèse d'origine, Paris, Éditions Galilée, « Incises », 1996, $135 \mathrm{p}$.

Séminaire sur l'hospitalité, troisième séance, II boîte., [Séminaire], 1996-1997 IMEC - Institut Mémoires de l'édition Contemporaine, 219 DRR 235.1.

Apories : mourir, s'attendre aux « limites de la vérité », Paris, Galilée, «Incises », 1995, 140 p.

La Voix et le phénomène: introduction au problème du signe dans la phénoménologie de Husserl, Paris, France, Presses universitaires de France, 1983, 117 p.

Jacques Derrida - La Différance, Éditions Ismael (1968), https://editions-ismael.com/ $\mathrm{fr} / 2016 / 05 / 27 / 1968$-jacques-derrida-la-differance-2, 1968, consulté le 31 octobre 2019.

, L'écriture et la différence, Paris, France, Éditions du Seuil, 1967, 435 p.

Kofman Sarah, Comment s'en sortir ?, Paris, Galilée, « Débats », 1983, 113 p.

Proust Françoise, «Impasses et passes », in Les cahiers du GRIF, $\mathrm{n}^{0} 1$, vol. 3, 1997, p. 5-10.

Rodrigues Carla, «Ce qui demeure irréductible dans le travail du deuil et dans la tâche du traducteur», in Revue ITER, $\mathrm{n}^{\circ}$ 2, vol. 1, 2020, « lire-travailler-derrida », p. 23.

Salanskis Jean-Michel, Derrida, Paris, France, les Belles lettres, 2010, $172 \mathrm{p}$.

Recebido em: $14 / 10 / 2020$

Aprovado em: 20/11/2020 